\title{
Intracranial Electroencephalography Reveals Selective Responses to Cognitive Stimuli in the Periventricular Heterotopias
}

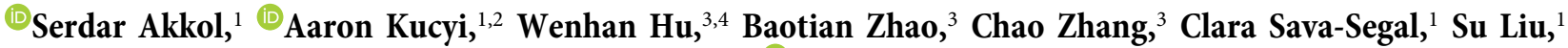 \\ Babak Razavi, ${ }^{1}$ Jianguo Zhang, ${ }^{3,4}$ Kai Zhang, ${ }^{3,4}$ and ${ }^{\circledR}$ Josef Parvizi $^{1}$ \\ ${ }^{1}$ Department of Neurology and Neurological Sciences, Stanford University, Stanford, California 94305, ${ }^{2}$ Department of Psychology, Northeastern \\ University, Boston, Massachusetts 02115, ${ }^{3}$ Department of Neurosurgery, Beijing Tiantan Hospital, Capital Medical University, Beijing 100070, \\ China, and ${ }^{4}$ Stereotactic and Functional Neurosurgery Laboratory, Beijing Neurosurgical Institute, Capital Medical University, Beijing 100070, China
}

Our recent work suggests that non-lesional epileptic brain tissue is capable of generating normal neurophysiological responses during cognitive tasks, which are then seized by ongoing pathologic epileptic activity. Here, we aim to extend the scope of our work to epileptic periventricular heterotopias (PVH) and examine whether the PVH tissue also exhibits normal neurophysiological responses and network-level integration with other non-lesional cortical regions. As part of routine clinical assessment, three adult patients with PVH underwent implantation of intracranial electrodes and participated in experimental cognitive tasks. We obtained simultaneous recordings from $\mathrm{PVH}$ and remote cortical sites during rest as well as controlled experimental conditions. In all three subjects (two females), cognitive experimental conditions evoked significant electrophysiological responses in discrete locations within the PVH tissue that were correlated with responses seen in non-epileptic cortical sites. Moreover, the responsive PVH sites exhibited correlated electrophysiological activity with responsive, non-lesional cortical sites during rest conditions. Taken together, our work clearly demonstrates that the PVH tissue may be functionally organized and it may be functionally integrated within cognitively engaged cortical networks despite its anatomic displacement during neurodevelopment.

Key words: periventricular heterotopia; sustained attention; high-frequency broadband; intracranial electroencephalography; intrinsic connectivity

Significance Statement

Periventricular heterotopias (PVH) are developmentally abnormal brain tissues that frequently cause epileptic seizures. In a rare opportunity to obtain direct electrophysiological recordings from $\mathrm{PVH}$, we were able to show that, contrary to common assumptions, PVH functional activity is similar to healthy cortical sites during a well-established cognitive task and exhibits clear resting state connectivity with the responsive cortical regions.

Received Nov. 2, 2020; revised Dec. 27, 2020; accepted Mar. 6, 2021.

Author contributions: S.A., A.K., J.Z., K.Z., J.P. designed research; S.A., A.K., W.H., B.Z., C.Z., C.S.S., B.R. performed research; S.A., A.K., B.Z., S.L. analyzed data; all authors wrote the paper.

This work was supported by the National Institute of Neurological Disorders and Stroke Grant R01NS078396, the National Institute of Mental Health Grant 1R01MH109954-01, and the National Science Foundation Grant BCS1358907 (to J.P.). Data collection from Beijing was supported by the National Natural Science Foundation of China Grants 81771399 and 81701276 . A.K. was supported by a Banting Fellowship from the Canadian Institutes of Health Research. We thank Jessica Schrouff and Pedro Pinheiro-Chagas for technical assistance with analysis.

The authors declare no competing financial interests.

Correspondence should be addressed to Josef Parvizi at jparvizi@stanford.edu or Kai Zhang at zhangkai62035@163.com.

https://doi.org/10.1523/JNEUROSCI.2785-20.2021

Copyright $\odot 2021$ the authors

\section{Introduction}

Periventricular heterotopia (PVH) is one of the most welldefined (Barkovich and Kjos, 1992; Barkovich and Kuzniecky, 2000) and commonly described (Barkovich and Kuzniecky, 2000; McRae et al., 2017) malformations of cortical development (MCDs; Barkovich, 2005). It is characterized by the failed migration of cortical neurons and the formation of nodular gray matter around the lateral ventricular walls. While clinical manifestations of PVH are diverse and range from global developmental delay to focal motor deficits, seizures are one of their most common features (Battaglia et al., 2006; Guerrini and Dobyns, 2014). Electrophysiological recordings in PVH have shown their direct involvement in generating seizures along with the overlying cortex, mostly (Li et al., 1997; Kothare et al., 1998; Aghakhani et al., 2005; Valton et al., 2008; Thompson et 
Table 1. Demographic, clinical, and behavioral performance information for three cases

\begin{tabular}{|c|c|c|c|}
\hline & Case 1 & Case 2 & Case 3 \\
\hline Age & 18 & 28 & 20 \\
\hline Gender & Female & Female & Male \\
\hline Handedness & Left & Right & Right \\
\hline Years of education & 11 years & 19 years & 13 years \\
\hline Duration of epilepsy & 2 years & 18 years & 8 years \\
\hline Treatment & $\begin{array}{l}\text { Medicine: oxcarbazepine } \\
\text { Surgery: radio frequency thermocoagulation } \\
\text { through depth electrodes after SEEG monitoring }\end{array}$ & $\begin{array}{l}\text { Medicine: oxcarbazepine, levetiracetam } \\
\text { Surgery: radio frequency thermocoagulation } \\
\text { through depth electrodes after SEEG monitoring }\end{array}$ & $\begin{array}{l}\text { Medicine: lamotrigine, zonisamide, and lacosamide } \\
\text { Surgery: responsive neurostimulation targeting } \\
\text { posterior locations }\end{array}$ \\
\hline Seizure types & Focal aware; focal aware to bilateral tonic-clonic & $\begin{array}{l}\text { Focal impaired awareness; focal impaired awareness } \\
\text { to bilateral tonic-clonic }\end{array}$ & Focal impaired awareness to bilateral tonic-clonic \\
\hline Video-EEG results & Right frontal lobe epileptic discharges & $\begin{array}{l}\text { Left frontal and anterior-middle temporal spikes } \\
\text { and irregular slow waves }\end{array}$ & $\begin{array}{l}\text { Right occipito-temporal onset seizure with right } \\
\text { posterior slowing of ongoing rhythm }\end{array}$ \\
\hline Behavioral results & $\begin{array}{l}\text { Average city-error rate of } 1.2 \% \text { (range: } 1-2 \% \text { ) and } \\
\text { average mountains-error rate of 19\% (range: } 7- \\
30 \% \text { ) }\end{array}$ & $\begin{array}{l}\text { Average city-error rate of } 1.1 \% \text { (range: } 1-1.2 \% \text { ) } \\
\text { and average mountains-error rate of } 13.3 \% \\
\text { (range: } 10-18 \% \text { ) }\end{array}$ & $\begin{array}{l}\text { Average city-error rate of } 4.2 \% \text { (range: } 2-5 \% \text { ) and } \\
\text { average mountains-error rate of } 58 \% \text { (range: } \\
40-70 \% \text { ) }\end{array}$ \\
\hline SEEG results & $\begin{array}{l}\text { Total of } 182 \text { SEEG electrodes, } 24 \text { within the PVH tissue. } \\
\text { Functionally active electrodes: } 7 \text { of } 24 \text { PVH, } 56 \text { of } \\
158 \text { non-PVH electrodes. } \\
\text { Epileptic sites within active sites: } 2 \text { of } 7 \text { active PVH } \\
\text { sites and } 17 \text { of } 56 \text { active non-PVH were epileptic. }\end{array}$ & $\begin{array}{l}\text { Total of } 144 \text { SEEG electrodes, } 26 \text { within the PVH tissue. } \\
\text { Functionally active electrodes: } 5 \text { of } 26 \text { PVH, } 3 \text { of } 118 \\
\text { non-PVH electrodes. } \\
\text { Epileptic sites within active sites: } 5 \text { of } 5 \text { active PVH } \\
\text { sites and } 3 \text { of } 3 \text { active non-PVH were epileptic. }\end{array}$ & $\begin{array}{l}\text { Total of } 140 \text { SEEG electrodes, } 38 \text { within the PVH tissue. } \\
\text { Functionally active electrodes: } 10 \text { of } 38 \text { PVH, } 16 \text { of } \\
102 \text { non-PVH electrodes. } \\
\text { Epileptic sites within active sites: } 1 \text { of } 10 \text { active PVH } \\
\text { sites and } 7 \text { of } 16 \text { active non-PVH were epileptic. }\end{array}$ \\
\hline
\end{tabular}

City-errors (or omission errors) are mistakes when a button press should be made but are missed; mountain-errors (or commission errors) are mistakes when a button press should be withheld but the button is pressed. Rate and range are given for the blocks done. PVH, periventricular heterotopia; EEG, electroencephalography; SEEG, stereo-EEG.

al., 2016; Pizzo et al., 2017), and treatment often involves the resection or ablation of this tissue ( $\mathrm{Li}$ et al., 1997; Aghakhani et al., 2005; Thompson et al., 2016; Mirandola et al., 2017). However, it remains largely unresolved whether PVH tissue maintains neurotypical sensorimotor and cognitive functions comparable to those seen in non-lesional brain tissue.

Besides few electrophysiological studies, previous functional neuroimaging studies have shown blood oxygenation level-dependent (BOLD) changes in the PVH sites similar to those seen in healthy subjects (Spreer et al., 2001; Kirschstein et al., 2003; Draganski et al., 2004; Villani et al., 2004; Danckert et al., 2007; Wagner et al., 2009; Christodoulou et al., 2012, 2013). However, it remains unknown to what extent these functional responses are localized within the PVH tissue, how the profile of neurophysiological responses in the PVH resembles the profile of activity in cortical sites, and whether the cortical and $\mathrm{PVH}$ responses are correlated during cognitive tasks and wakeful rest. We aimed to address this gap of knowledge by using intracranial electroencephalography (iEEG) recordings simultaneously from the PVH and cortical sites in patients with known focal epilepsies. This method offers a sensitive and direct means by which one can investigate neuronal population activity within and across brain sites during specific cognitive tasks with millimeter and millisecond precision (Parvizi and Kastner, 2018).

We recently reported that non-lesional epileptic brain tissue is capable of generating normal physiological responses during cognitive tasks (Liu and Parvizi, 2019). Here, we aim to extend the scope of our prior work to epileptic PVH lesional tissue (defined clinically) and examine its capability to generate normal neurophysiological responses as well as network-level integration with other functionally responsive brain regions. Inspired by prior non-invasive studies of $\mathrm{PVH}$, we designed the current study to address the following three aims. First, to offer neurophysiological validation of non-invasive neuroimaging findings showing cognitive task-evoked responses in the PVH that are comparable to those in non-epileptic cortical sites; Second, to explore with high anatomic precision whether the PVH tissue shows homogenous profiles of task-evoked responses or whether specific functions are "localized" to specific sites within the $\mathrm{PVH}$ tissue; and third, to test whether there is spontaneous electrophysiological coupling between the PVH tissue and responsive cortical sites during a rest condition and whether this coupling is different for epileptic and non-epileptic PVH sites.

The overarching goal of the study was to address a core question namely to what extent a structurally aberrant and seemingly pathologic brain tissue (such as $\mathrm{PVH}$ ) may still carry physiological functional properties. The rationale for our current study was to test the hypothesis that cognitive functions are preserved in heterotopic tissue. While our observations provide a platform for testing this hypothesis, our sample of three exemplary patients precludes major generalizations about all heterotopic tissue and how it contributes to the brain's normal functions. However, knowledge in this domain will help us generate hypotheses about the position of pathologic tissue such as $\mathrm{PVH}$ within the physiological architecture of the brain.

\section{Materials and Methods}

\section{Subjects}

Here, we describe three cases (two females, one male) with medically refractory epilepsy and $\mathrm{PVH}$ who underwent neurosurgical treatment. Cases 1 and 2 were collected at Beijing Tiantan Hospital, and case 3 was collected at Stanford University Medical Center (for full demographic and clinical information, see Table 1). Patients were monitored for 7$40 \mathrm{~d}$ with iEEG as part of their presurgical evaluation to define epileptogenic zones ( $40 \mathrm{~d}$ for case $1,27 \mathrm{~d}$ for case 2 , and $7 \mathrm{~d}$ for case 3 ). Patients were implanted with depth electrodes (stereo-EEG; SEEG) that were placed stereotactically within one or both hemispheres. Electrode placement was decided based on clinical needs. All of the patients provided verbal and written consent to participate in research, and either the Medical Ethics Committee of Beijing Tiantan Hospital (cases 1 and 2) or the Stanford Institutional Review Board (case 3) approved all procedures described herein.

\section{Experimental design and statistical tests}

Neuroimaging

All three cases underwent a preoperative structural MRI (T1-weighted) and a postoperative computed tomography (CT) scan after electrode implantation. In Beijing cases, a 3.0 Tesla Siemens MAGNETOM Verio system with a 32-channel head coil was used. For neuroimaging at 
Stanford, a 3.0 Tesla GE 750 MR system equipped with an 8-channel receive only head coil (8HRBrain) was used. We used the iELVis toolbox (Groppe et al., 2017) for anatomic localization of electrode contacts. First, we processed and reconstructed the T1 scan using Freesurfer v6.0.0 (recon-all command; Fischl et al., 1999). Then, we aligned the postimplant CT image to the structural MRI using BioImage Suite (Papademetris et al., 2006) to manually label each electrode contact on the T1-registered CT image. Electrode locations within PVH sites were confirmed visually by the patients' epileptologists (K.Z. and J.P.) on postoperative CT scans aligned to preoperative MRI.

\section{SEEG data acquisition}

For all cases, data were recorded via a clinical monitoring system (Nihon Kohden) with a sampling rate of $1000 \mathrm{~Hz}$ and a bandpass filter of $0.08-$ $300 \mathrm{~Hz}$ for all task sessions. Based on clinical need, patients were implanted with 14 SEEG electrodes with 182 contacts to left hemisphere, 11 SEEG electrodes with 144 contacts to right hemisphere, or 14 SEEG electrodes with 140 contacts to both hemispheres, respectively (Table 1). For cases 1 and 2 (Beijing), depth electrode contacts (HKHS Healthcare) had a contact length of $2 \mathrm{~mm}$, diameter of $0.8 \mathrm{~mm}$, and interelectrode spacing of $1.5 \mathrm{~mm}$. For case 3 (Stanford), SEEG electrodes (Ad-Tech Medical Instrument Corporation) were cylindrically-shaped $(0.86-\mathrm{mm}$ diameter, 2.29-mm height) and with interelectrode spacing of 5-10 mm. During recordings, the SEEG signals were referenced to the most electrographically silent electrodes outside of the seizure focus.

\section{Cognitive task performance}

Patients performed a well-described sustained attention task, the gradual-onset continuous performance task (gradCPT), as employed in prior fMRI and iEEG studies (Esterman et al., 2013, 2014; Rosenberg et al., 2013; Fortenbaugh et al., 2018; Kucyi et al., 2020; Kucyi and Parvizi, 2020). Data were collected on a laptop computer that was placed in front of patients at bedside around $70 \mathrm{~cm}$ from the subjects' eyes at chest level. During task performance, grayscale visual images of either city or mountain scenes appeared within round frames (on a white background) and gradually transitioned from one to another for the duration of the task. Using linear pixel-by-pixel interpolation within each trial, image coherence began gradually increasing from time 0 (minimum coherence) until $400 \mathrm{~ms}$ (maximum coherence) and then gradually decreasing back to minimum coherence (at $800 \mathrm{~ms}$ ) for a total interstimulus interval of $800 \mathrm{~ms}$. Scenes were presented randomly with $10 \%$ mountain and $90 \%$ city stimuli, but the same scene could not repeat on consecutive trials. Subjects were instructed to respond by pressing the spacebar key on the laptop computer for city events (frequent category), but not for mountain events (infrequent category). Each task session lasted $6 \mathrm{~min}$, and during this time, subjects were asked to perform as accurately and as quickly as possible. Patients performed five, six, and five sessions, respectively. Figure $1 A$ shows the general structure of the task.

\section{SEEG processing}

Data were processed with a previously described pipeline (Kucyi et al., 2020). Each SEEG run was preprocessed individually using publicly available tools [SPM12 (Kiebel and Friston, 2004) and Fieldtrip (Oostenveld et al., 2011)] with a MATLAB pipeline (R2018a, MathWorks) that was developed in-house (https://github.com/JessicaSchrouff/ECoG_preprocessing SPM). First, notch filtering was performed to remove line noise and its harmonics (specifically, of $60 \mathrm{~Hz}$ for Stanford and of $50 \mathrm{~Hz}$ for Beijing with zero-phase, third order, Butterworth filter with band-stop between 57-63, $117-123$, and $177-183 \mathrm{~Hz}$ for data from Stanford, and band-stop between $47-53,97-103$, and $147-153 \mathrm{~Hz}$ for data from Beijing). We then performed common average re-referencing, excluding electrodes that either (1) showed pathologic activity during clinical monitoring (as noted by a neurologist); (2) had a five-times above or below the median variance across all electrodes; or (3) contained automatically detected spikes (i.e., had more than three times the median number of spikes across all electrodes, with spikes defined as $100-\mu \mathrm{V}$ changes between successive samples).

Following re-referencing, we performed time-frequency decomposition to estimate the power spectra, using a Morlet transform with five wavelet cycles and with frequencies of interest log-spaced between 1 and
$170 \mathrm{~Hz}$ (38 total values). The decomposition was then rescaled by the log ratio to normalize the distributions of power amplitude estimates at each frequency of interest. We then averaged the power amplitude estimates (envelopes) within the high-frequency broadband (HFB; 70-170 Hz), which is a well-established reflection of the BOLD signal and neuronal population spiking (Parvizi and Kastner, 2018; Watson et al., 2018).

As in prior work (Kucyi et al., 2020), to assess the statistical significance of HFB responses during the 0 - to $1500-\mathrm{ms}$ period (where time 0 is minimum gradCPT image coherence), we adopted a nonparametric cluster-based permutation test [using Fieldtrip (Maris and Oostenveld, 2007) functions] conducted separately for all electrodes within each subject and statistically correcting for multiple electrodes separately across the three different conditions: (1) commission error (CE): errors on mountains; (2) correct omissions (CO), correct responses on mountains; (3) correct commission (CC), correct presses during cities. Conditions were defined as follows: mountain-error (or CE) was when the subject should have withheld pressing but erroneously pressed the button; mountain-correct (or $\mathrm{CO}$ ) was when the subject should have withheld and did withhold; cities-correct (or CC) was when the subject should have pressed and did press. For each pair of comparisons, we first performed a two-tailed $t$ test and adjacent samples exceeding the threshold of $p=0.05$ were grouped together into clusters. The sum of $t$ values within each cluster was calculated for cluster-level statistics, and the maximum of these values was taken as the test statistic. These procedures were then repeated using the Monte Carlo method with 10,000 permuted randomizations of trial type assignments. Electrodes that included observed clusters with a Monte Carlo significance probability $<0.05$ were deemed statistically significant. PVH electrodes which showed significance in at least one of these comparisons were selected for the intrinsic connectivity analysis see Resting state and task related intrinsic connectivity analysis

To further investigate the network-level integration of active PVH sites dynamically, HFB power amplitude from each mountain-error trials (0-1200 ms locked to mountain image onset) was normalized to its maximum and then averaged. Since there were not enough trials to accurately compute response onset latency, here we only present these results descriptively to illustrate near-simultaneous activations in active PVH sites. We also examined the dynamics of the integration across the trials with averaging the HFB power amplitude of the time period 400-1200 ms of mountain (infrequent) image onset for PVH and other cortical sites.

\section{Characterization of pathologic tissue}

We characterized a recording electrode site as part of the epileptic network on the basis of two criteria: (1) the clinical epilepsy team considered the site either as the ictal onset zone or involved early in the propagation of seizures; and (2) the site showed clearly pathologic highfrequency oscillations (HFOs) that are known to be a reliable marker for epileptic zone (Jacobs et al., 2010; Liu et al., 2018; Liu and Parvizi, 2019). The ictal onset channels were visually identified by two senior epileptologists independently who reviewed and labeled the channels with the earliest ictal discharges during recorded seizures. These reviews were performed by the clinical teams before and independent from this study. Clinicians defined "ictal events" as "rhythmic and evolving epileptiform activity with or without low amplitude high-frequency activity, which were clearly distinguishable from background activity." Propagation of seizures was defined as clear ictal discharge pattern identified on channels following earliest onset. HFOs were identified in EEG recordings obtained randomly from interictal states (average $36 \mathrm{~min}$ ). Resting state recordings were at least $4 \mathrm{~h}$ before or after any ictal event. We implemented an unsupervised method that has been previously validated for the quantitative identification of pathologic HFOs (Liu et al., 2016, 2018; Liu and Parvizi, 2019) that are distinct from epileptic spikes in neural recordings. The method was established on the broadly accepted definition of spontaneous interictal HFO, namely, a transient oscillatory activity possessing a minimum of four oscillatory cycles that are clearly distinct from background activities (Worrell et al., 2012). The identified HFOs exhibited abrupt and transient power increase in the high-band (recognized as an isolated light "blob" over $80 \mathrm{~Hz}$ in the time-frequency 
A

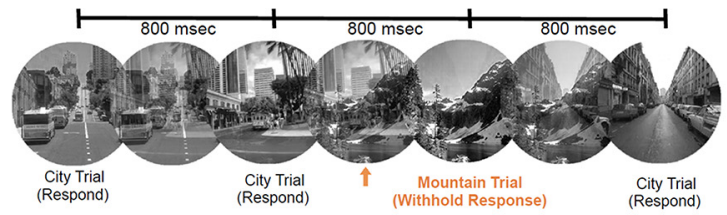

B Case-1
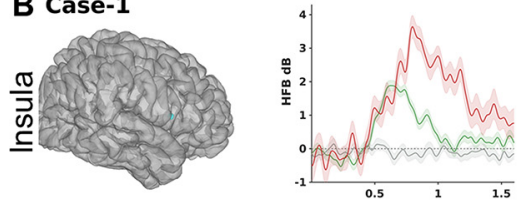

$\sum_{\substack{D \\ d}}^{T}$

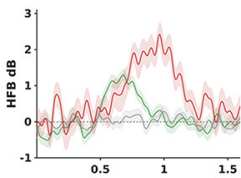

$\sum_{\stackrel{D}{D}}^{T}$ है।
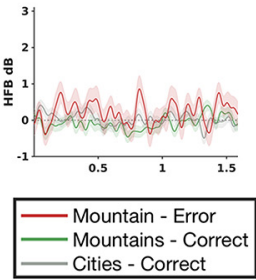

\section{Case-2}
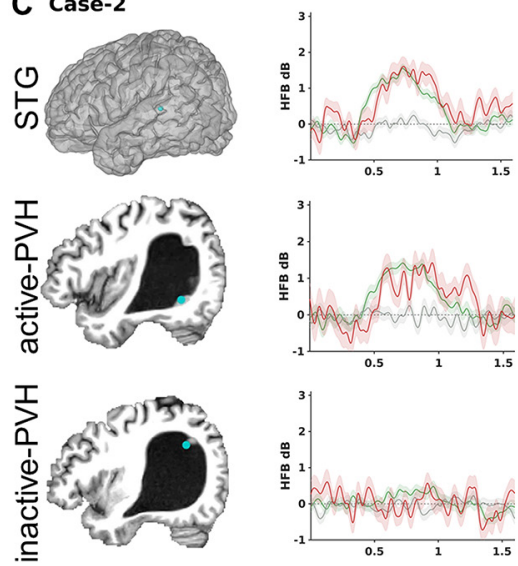

D Case-3
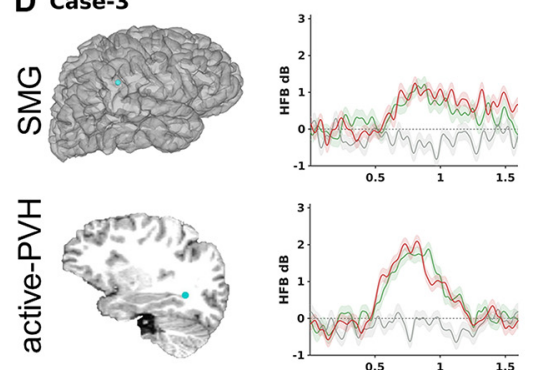

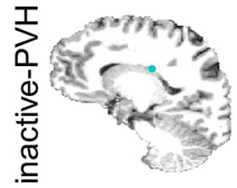

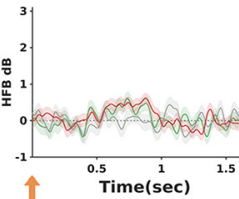

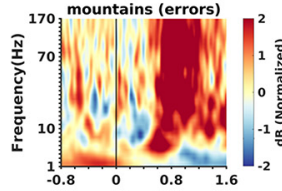
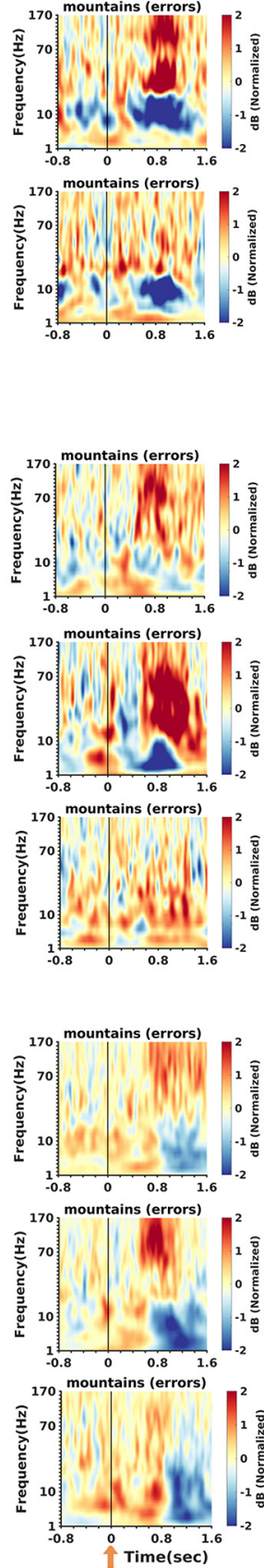
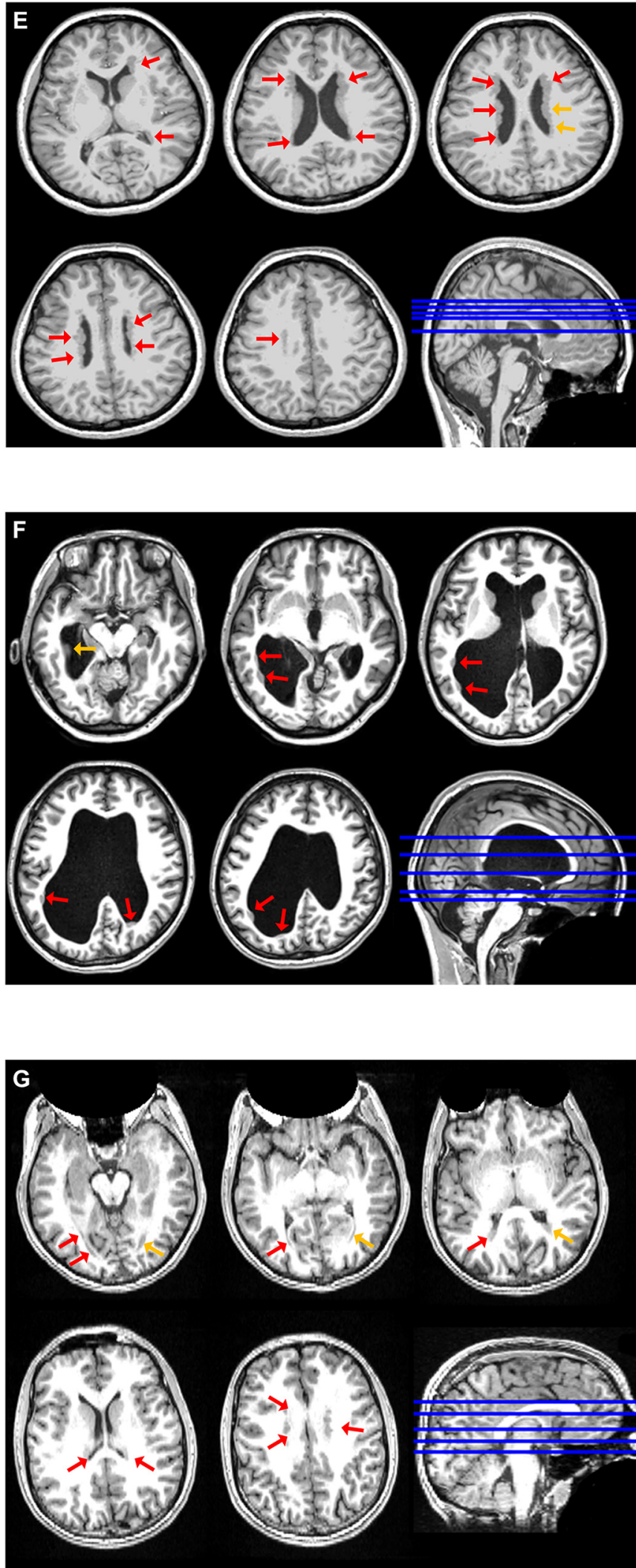

Figure 1. Task description, details from example sites and coverage and the extent of PVH. $\boldsymbol{A}$, The GradCPT paradigm. City and mountain scene images faded in and out continuously from one image to the next every $800 \mathrm{~ms}$. $\boldsymbol{B}-\boldsymbol{D}$, Three electrodes (one non-epileptic, one active PVH, one inactive PVH) from each case with their locations (in sagittal section or 3D image), HFB power amplitude (in decibels, normalized) and spectrograms of mean of mountains-error trials (orange arrows show onset of trials, time 0 ). $\boldsymbol{E}-\boldsymbol{G}$, Axial MR images and their levels in mid-sagittal image for each case. Red and orange arrows locate PVH tissues and orange arrows show the treatment sites after SEEG monitoring. STG, superior temporal gyrus; SMG, supramarginal gyrus. 
map), known as one of the spectral properties making HFOs distinct from spikes. The approach contains three main stages depicted as follow: in the first stage, after bandpass filtering the data between 80 and $450 \mathrm{~Hz}$, all anomalies were detected and extracted through an amplitude-based initial detector and sieved according to a series of HFOselective criteria. Next, HFO-distinguishing features were extracted from the time-frequency maps of all candidate events. These features were used with Gaussian mixture model clustering to isolate HFOs from interictal spikes (with residual energy in the high-band) and other arbitrary activities. Finally, we applied density-based spatial clustering (DBSCAN) with varying cluster radius and computed the corresponding number of sub-clusters to estimate the waveform similarity of detected HFO activities. The area-under-curve of the "clustering ratio curve" (i.e., the change in the number of sub-clusters with respect to different radius values) calculated from the detected HFOs was compared with the published data characterizing the pathologic HFOs generated from the seizure onset zone. The detected HFOs presented repetitive waveform patterns that formed compact and inhomogeneous clusters in the original high-dimensional space of the raw time series, showing consistency with the stereotyped waveform patterns that characterize pathologic HFOs specifically indicative to seizure onset zones.

\section{Resting state and task related intrinsic connectivity analysis}

For the interelectrode correlation analysis, we used both task and resting state (details in previous sections) HFB signals filtered to the 0.1 - to $1-\mathrm{Hz}$ range, as done previously for iEEG connectivity analysis (Keller et al., 2013; Kucyi et al., 2018). Our goals were (1) to get a global sense of which non-epileptic tissue electrodes had similar activation patterns as the PVH electrodes during task and (2) to test whether all significantly active PVH electrodes had similar activation patterns. To do so, we used the envelope of HFB signal during task sessions to correlate activities of PVH "seed" electrodes with "target" electrodes located within non-epileptic functioning tissue (while excluding electrodes in the PVH and neighboring electrodes to the seed). It has been previously shown that the low-frequency envelope of HFB signal is temporally correlated with populationlevel spiking activity and and spatially correlated with fMRI-BOLD intrinsic network topography (Nir et al., 2008; Manning et al., 2009; Keller et al., 2013; Foster et al., 2015).

In order to analyze possible intrinsic connectivity during resting state, we used the same approach for activity during rest sessions. Before proceeding, we manually checked both the raw and HFB signal from each session and further excluded electrodes from that session that had not been excluded with the automated pipeline, but still showed any salient discontinuities in either of the signals. Subsequently, a bandpass temporal filter (zero-phase, Butterworth, fourth order) was applied to the unsmoothed HFB signal, retaining frequencies between 0.1 and $1 \mathrm{~Hz}$, as done previously (Kucyi et al., 2020).

Using this low-frequency HFB signal, we performed a pair-wise correlation between electrodes and obtained $r$ values between electrodes for each run. To be able to estimate the mean correlation value of all sessions, these $r$ values were transformed into $z$ values using Fisher's ztransform. After this step, we averaged $z$ values for each pair from all sessions. In order to understand whether all PVH electrodes have similar activation patterns during task performance, we calculated the top $5 \%$ of averaged $z$ values of significantly active PVH electrodes with non-epileptic tissue electrodes. To do so, we excluded $z$ values from other PVH electrodes and electrodes within $13 \mathrm{~mm}$ (Euclidean distance) to find non-epileptic sites that were not in close proximity of one another. This distance excludes the possible presence of HFB activity arising from common neuronal populations measured by neighboring electrodes; such effects were measured to be within $<1 \mathrm{~cm}$ in animal studies (Engel et al., 1990; Katzner et al., 2009). By doing so, we were able to map the differences in activity across the PVH tissue. We adopted this same correlation analysis for rest sessions to understand whether this connectivity pattern remained consistent during rest or whether activity in the PVH dissociates from non-epileptic networks during rest. We then correlated the $z$-scored $r$ values to determine consistency in neural activity across task and rest.

\section{Results}

Patient characteristics and behavioral performance

As in previous studies of PVH (Battaglia et al., 2006; Guerrini and Dobyns, 2014), the cases described here started having seizures within the first two decades of their lives. Patient histories, semiological details, preoperative evaluation results and preoperative and postoperative treatment details are presented in Table 1. The extent of the PVH is as following: case 1, bilateral around the body and the tips of frontal and occipital of the lateral ventricles; case 2, bilateral (left more than right) scattered focal nodular PVH tissues around the enlarged atrium and the occipital horn of lateral ventricles; case 3 , bilateral around the body and extending from the tips of occipital horns of lateral ventricles (see Fig. $1 E-G)$. We recorded from a total of 466 electrode contacts in three cases, including 88 contacts in PVH tissue and 30 of 88 $\mathrm{PVH}$ sites being epileptic. We defined epileptic areas with following criteria: being in the seizure onset zone or showing epileptiform spikes revealed by clinical video-EEG, and showing HFOs (see Materials and Methods). After SEEG monitoring, case 1 and case 2 underwent thermocoagulation and case 3 was implanted with responsive neurostimulation. Treatment sites were shown with orange arrows in Figure $1 E-G$.

The gradCPT, used here, has been validated by several groups, including our own group, on both healthy and clinical populations (Rosenberg et al., 2013; Esterman et al., 2014; DeGutis et al., 2015; Fortenbaugh et al., 2015; Kucyi et al., 2020). During this task, subjects are instructed to withhold responses to unique visual stimuli (pictures of mountains) while pressing the key button for frequent images of cities. Fortenbaugh et al. (2015) showed that the rate of erroneous responses during infrequent mountain images (i.e., mountains-error or $\mathrm{CE}$ ) is around $10-70 \%$ and average rate of erroneous responses to city images (city-error or omission error) is around $1-10 \%$ in a populationlevel study of 10,000 subjects . As seen in Table 1, the rates of omission and commission errors in our clinical subjects were comparable to those previously seen in typically-developing subjects.

\section{Anatomically localized functional responses}

We found iEEG evidence for task-evoked responses in the cortical sites during experimental condition of sustained attention and continuous task performance. While our sampling of the brain was sparse to begin with, we found selective activations only in few but not all cortical sites being sampled (Table 1; Fig. 1). These sites were located in the dorsal anterior insular cortex, dorsolateral prefrontal cortex, superior-middle-inferior temporal gyri, lateral occipital cortex, posterior cingulate cortex, hippocampus, and precentral gyrus.

More importantly, single subject level analysis of dense recordings from the $\mathrm{PVH}$ tissue revealed that these responses were found, also with a sparse distribution, within the PVH itself. In all three patients, we identified electrodes in the PVH tissue that showed significant (Monte Carlo, $p<0.05$ corrected) taskevoked activations (Table 1; Fig. 1). Within each patient, PVH activations during task performance were temporally and spectrally similar to those seen in select cortical areas (Fig. 1). Figure $1 E-G$ shows five axial MR images and their levels in mid-sagittal image to depict the extent of PVH and the treatment sites. In 

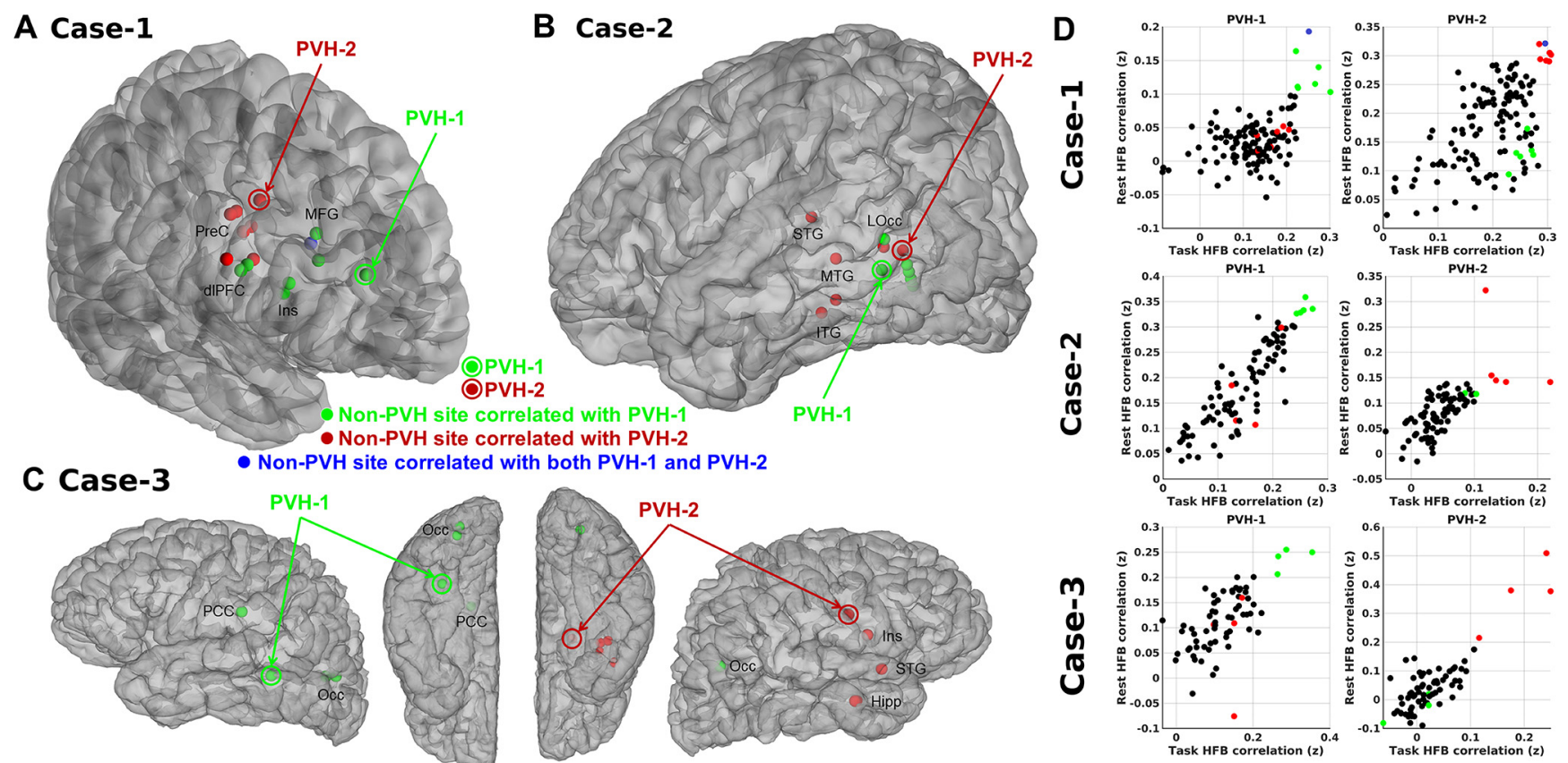

Figure 2. Intrinsic connectivity results. $\boldsymbol{A}-\boldsymbol{C}$, Semi-transparent $3 \mathrm{D}$ images of each case, respectively. $\boldsymbol{D}$, Correlation plots of task and rest $z$ scores. Each plot represents the corresponding electrode from $\boldsymbol{A}-\boldsymbol{C}$. Each dot represents a non-epileptic healthy brain site and the red, green, and yellow dots correspond to the sites of the same color that are shown in panels $\boldsymbol{A}-\boldsymbol{C}$. Correlations of z-scored $r$ values from task and rest correlations: $0.46,0.57,0.87,0.67,0.64$, and 0.84 from left to right and top to bottom. Colored arrows point to the selected PVH sites. PreC, precentral gyrus; MFG, middle frontal gyrus; dIPFC, dorsolateral prefrontal cortex; Ins, insula; STG-MTG-ITG, superior-middle-inferior temporal gyri; LOcc, lateral occipital; PCC, posterior cingulate cortex; Occ, occipital; Hipp, hippocampus.

case 1 and case 2, while there were bilateral PVH tissues, videoEEG findings showed unilateral onset, thus requiring unilateral implantation.

Task-activated PVH and non-PVH sites had similar probabilities of being epileptic (all cases combined: eight of 22 active PVH electrodes and 27 of 75 active non-PVH electrodes were defined as pathologic given the presence of epileptic spikes or seizures and pathologic HFOs ( $\chi^{2}$ statistic was $\left.0.001, p=0.98\right)$.

To eliminate the possibility of volume conduction from surrounding tissue into the $\mathrm{PVH}$, we carefully examined the distances between each active PVH electrode and found no volume conduction from other responsive electrodes. For example, while the electrode shown in the middle row of Figure $1 B$ had significant activations, the most adjacent electrodes within the PVH did not show significant activation during the task (data not shown).

\section{Functional coupling between PVH and cortical sites}

We next investigated whether the PVH sites that were functionally responsive were coupled with functionally responsive cortical sites during both task and rest conditions. Showing resting state coupling would suggest that the task coupled sites are also intrinsically connected (van den Heuvel and Hulshoff Pol, 2010; Kucyi et al., 2018). For this analysis, we correlated the activity of $\mathrm{PVH}$ sites with that of all other (non-neighboring) electrodes during both task and resting state conditions. During task performance, we found that activated PVH sites had highly correlated activity with some activated cortical sites (Fig. 2). Critically, PVH functional connectivity during the task state was highly correlated with PVH functional connectivity during the resting state, suggesting intrinsic connectivity ( $r$ values ranged from 0.46 to 0.84 ; all $p<0.01$, FDR-corrected).

Additional evidence for the functional activity in PVH sites was found in the dynamics of task-evoked activity compared with task-active non-epileptic cortical sites. We found that PVH sites responded with the same profile as the cortical sites (Fig. 3). PVH responses could be simultaneous, before, or after cortical activations. When we looked across trials, the average taskevoked HFB power amplitude [averaged in 400-1200 ms of mountain (infrequent) image onset] for $\mathrm{PVH}$ and other cortical sites were highly correlated ( $r$ values of seed-based connectivity during task sessions: 0.35 and $0.07, p<0.05)$. These results clearly illustrate task evoked network connectivity between the PVH and select cortical sites.

Finally, we tested whether epileptic PVH sites had exclusive task-based or resting-state coupling with only epileptic cortical sites. We are mindful that invasive EEG approach suffers from the problem of sparse sampling which makes it less suitable for network mapping. Therefore, the purpose of our analysis was not to provide a comprehensive map of PVH connectivity with the cerebral cortex. Instead, we solely were interested in probing whether the epileptic PVH sites were only connected with epileptic cortical sites. Our analysis revealed no exclusive pattern of connectivity. Eight of 22 PVH sites were epileptic and functionally responsive. These sites showed task-evoked coupling with 27 epileptic and 16 non-epileptic cortical sites. By comparison, 14 task-active PVH sites were confirmed to be non-epileptic and these were connected with 25 epileptic and 42 non-epileptic cortical sites ( $\chi^{2}$ statistic 6.82, $p=0.009$ ).

\section{Discussion}

Here, using direct recordings of neuronal population activity, we demonstrated that there is functional heterogeneity within $\mathrm{PVH}$ tissue. Task-active sites in the PVH are functionally correlated with remote cortical sites some of which are clearly void of any epileptiform activity. Moreover, PVH sites maintain correlated activity with functional cortical sites during task and resting states, suggesting intrinsic coupling. 

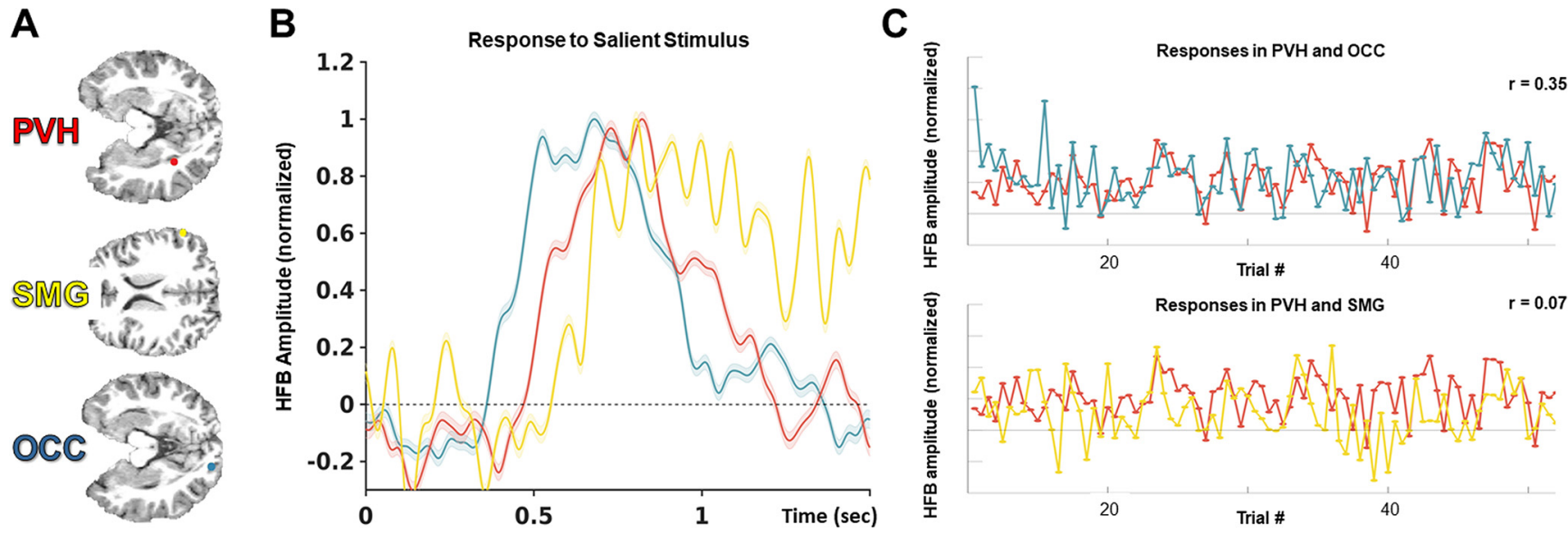

Figure 3. Dynamics of activity in PVH and cortical sites during task. $A$, Locations of the recordings from case 3 are PVH, occipital (Occ) and supramarginal gyrus (SMG). $B$, Average ( \pm SEM in decibels) HFB power amplitude (normalized to its maximum from each trial) in one PVH and two non-epileptic cortical sites from case 3 during CE trials. C, Average HFB power amplitude for each mountain trial (from 400 to 1200 ms of mountain image onset, not normalized to its maximum) shows that PVH was active during the task similar to non-epileptic active cortical sites; $r$ values indicate the results of seed-based connectivity of two sites $(p<0.05)$.

Besides neuroimaging evidence, there are prior SEEG reports suggesting potential involvement of PVH in cognitive processes (Kirschstein et al., 2003; Wagner et al., 2009). For instance, Wagner et al. (2009) reported two patients in whom stimulation of PVH tissue caused acoustic or visual hallucinations, and Kirschstein et al. (2003) found focal ERPs generated within the PVH lesion in seven out of eight patients during continuous visual word recognition task. Our current study further showed that task-evoked HFB response in PVH, highly similar to canonical responses found in non-lesional tissue (Crone et al., 2006), is well localized and diverse through the PVH tissues, with strong replications found in 3 cases using a well-established cognitive task.

Though PVH tissue is located abnormally inside the white matter, our findings suggest that portions of this atypical tissue exhibit normal physiological responses to cognitive stimuli and possess intrinsic connectivity with non-epileptic cortical sites. Moreover, PVH tissue seems to be functionally heterogeneous, and we posit that different sites within the PVH have selective neuroanatomical connections with cortical and other subcortical functional regions. In other words, separate "areas" might exist within the PVH tissue. While functional neuronal ensembles within the PVH did not migrate properly, they still developed and retained their functional place within widespread cortical networks. In keeping with this, extant evidence suggests discrete structural and functional connectivity between $\mathrm{PVH}$ and certain areas of the cerebral cortex (Kakita et al., 2002; Christodoulou et al., 2012; Liu et al., 2017). Prior works have focused specifically on the involvement of the PVH tissue in epileptic networks (Valton et al., 2008; Pizzo et al., 2017). Pizzo et al. (2017) used their epileptogenicity index which relies on signal energy redistribution from lower toward higher frequency bands and delays in appearance of high-frequency components in a given structure with respect to the first structure involved in "seizure onset." By relying on this index, they quantified the level of epileptogeneicity of PVH and nearby cortical sites. Valton et al. (2008) studied three seizures in a single patient and reported "interdependencies" (i.e., correlation of signals) in two seizures involving both heterotopia and cortical structures. They also found that the stimulations of heterotopic tissue induced responses in remote cortical structures. In this study, however, of three seizures reported, clearly local seizures were seen with onset in the heterotopic tissue without significant interaction with the cortical tissue. The profile of such network connectivity across PVH and the cerebral cortex could not be examined in our current study given the sparse recordings typical for iEEG approach and also relatively small number of subjects recruited in our study. However, our findings indirectly support the hypothesis that the epileptic PVH tissue might be preferentially (but not exclusively) connected with epileptic, rather than non-epileptic, cortical sites (Aghakhani et al., 2005; Tassi et al., 2005).

The cognitive task used in this report, gradCPT is an experimental paradigm for testing attention and response control. It has been extensively validated in the literature including our own studies of epilepsy patients performing this experiment (Esterman et al., 2014; Fortenbaugh et al., 2015; Kucyi et al., 2020; Kucyi and Parvizi, 2020). Behavioral performance of the participants in our current study was comparable to those reported in the literature. Additionally, all three patients recruited in the current study were highly educated [either in college (case 1 and case 3) or with a graduate degree (case 2)]. These findings confirm that cases in our sample had a functional cognitive capacity that is comparable to healthy populations. Furthermore, all cases had activations in non-epileptic cortical regions (Fig. 1) that have been shown by neuroimaging studies of normal healthy subjects and our own studies of epilepsy patients to be engaged during this task (Kucyi et al., 2020). More strikingly, activations in the epileptic PVH recording sites were similar to those in non-epileptic cortical sites. Several observations helped us to reach this conclusion. (1) In a recent work, we have shown that there are different classes of high-frequency activity (Liu and Parvizi, 2019). The spectrogram profiles shown in Figure 1 are highly consistent with "physiological" HFB that we showed previously (i.e., based on broad band and response duration/profile; Kucyi et al., 2020; Kucyi and Parvizi, 2020). (2) The HFB activations in PVH were time-locked to task-related cognitive events known to be associated with physiological responses within multiple brain regions/networks (Kucyi et al., 2020). (3) The timing and spectral properties of HFB activations were similar for healthy tissue versus PVH tissue.

Our study was limited by several factors. First, our cohort included only three patients with PVH who had also participated in experimental tasks. To mitigate the possible confounding effect of small number of observations, all our analyses were performed at the level of each single individual. As we have shown 
before, given the excellent signal-to-noise ratio of iEEG signal, single-subject level inferences are highly valuable when the observed effect is documented with high fidelity within and across individuals (Foster et al., 2015; Kucyi and Parvizi, 2020). Second limitation of our study pertains to use of different iEEG recordings across the two medical centers (Beijing and Stanford). However, our previous work suggests similar task-evoked electrophysiological response profiles across centers for the gradCPT (Kucyi et al., 2020) and here we were able to capture similar task evoked electrophysiological responses across the two centers (Table 1). Lastly, we acknowledge that deciding on the pathologic status of a tissue may be partly subjective in the clinical practice with possible interrater variability. To mitigate this risk, we not only relied on clinical observations but also added a clearly objective criterion namely automatic detection of pathologic (and not physiological) HFO using previously validated parameters.

In closing, our current study builds on our recent findings that MRI-negative but electrophysiologically epileptic structures are capable to generate normal physiological responses (Liu and Parvizi, 2019). Here, we extend our prior findings to MRI-positive and seemingly pathologic tissue such as $\mathrm{PVH}$ which is often considered as a pathologically displaced tissue. To conclude, $\mathrm{PVH}$ are integrated into functional cortical circuits and, hence, this not only can explain how they contribute the epileptogenicity but how they can also contribute to physiological cortical functions.

\section{References}

Aghakhani Y, Kinay D, Gotman J, Soualmi L, Andermann F, Olivier A, Dubeau F (2005) The role of periventricular nodular heterotopia in epileptogenesis. Brain 128:641-651.

Barkovich AJ (2005) Malformations of cortical development. In: Magnetic resonance in epilepsy (Kuzniecky RI, Jackson GD, eds), Ed 2, pp 221248. Burlington: Elsevier Academic.

Barkovich AJ, Kjos BO (1992) Gray matter heterotopias: MR characteristics and correlation with developmental and neurologic manifestations. Radiology 182:493-499.

Barkovich AJ, Kuzniecky RI (2000) Gray matter heterotopia. Neurology 55:1603-1608.

Battaglia G, Chiapparini L, Franceschetti S, Freri E, Tassi L, Bassanini S, Villani F, Spreafico R, D’Incerti L, Granata T (2006) Periventricular nodular heterotopia: classification, epileptic history, and genesis of epileptic discharges. Epilepsia 47:86-97.

Christodoulou JA, Walker LM, Del Tufo SN, Katzir T, Gabrieli JDE, Whitfield-Gabrieli S, Chang BS (2012) Abnormal structural and functional brain connectivity in gray matter heterotopia. Epilepsia 53:10241032.

Christodoulou JA, Barnard ME, Del Tufo SN, Katzir T, Whitfield-Gabrieli S, Gabrieli JDE, Chang BS (2013) Integration of gray matter nodules into functional cortical circuits in periventricular heterotopia. Epilepsy Behav 29:400-406.

Crone NE, Sinai A, Korzeniewska A (2006) High-frequency gamma oscillations and human brain mapping with electrocorticography. Prog Brain Res 159:275-295.

Danckert J, Mirsattari SM, Bihari F, Danckert S, Allman A-A, Janzen L (2007) Functional MRI characteristics of a focal region of cortical malformation not associated with seizure onset. Epilepsy Behav 10:615-625.

DeGutis J, Esterman M, McCulloch B, Rosenblatt A, Milberg W, McGlinchey R (2015) Posttraumatic psychological symptoms are associated with reduced inhibitory control, not general executive dysfunction. J Int Neuropsychol Soc 21:342-352.

Draganski B, Winkler J, Flügel D, May A (2004) Selective activation of ectopic grey matter during motor task. Neuroreport 15:251-253.

Engel AK, König P, Gray CM, Singer W (1990) Stimulus-dependent neuronal oscillations in cat visual cortex: inter-columnar interaction as determined by cross-correlation analysis. Eur J Neurosci 2:588-606.
Esterman M, Noonan SK, Rosenberg M, DeGutis J (2013) In the zone or zoning out? Tracking behavioral and neural fluctuations during sustained attention. Cereb Cortex 23:2712-2723.

Esterman M, Rosenberg MD, Noonan SK (2014) Intrinsic fluctuations in sustained attention and distractor processing. J Neurosci 34:1724-1730.

Fischl B, Sereno MI, Dale AM (1999) Cortical surface-based analysis. II: inflation, flattening, and a surface-based coordinate system. Neuroimage 9:195-207.

Fortenbaugh FC, DeGutis J, Germine L, Wilmer JB, Grosso M, Russo K, Esterman M (2015) Sustained attention across the life span in a sample of 10,000: dissociating ability and strategy. Psychol Sci 26:1497-1510.

Fortenbaugh FC, Rothlein D, McGlinchey R, DeGutis J, Esterman M (2018) Tracking behavioral and neural fluctuations during sustained attention: a robust replication and extension. Neuroimage 171:148-164.

Foster BL, Rangarajan V, Shirer WR, Parvizi J (2015) Intrinsic and task-dependent coupling of neuronal population activity in human parietal cortex. Neuron 86:578-590.

Groppe DM, Bickel S, Dykstra AR, Wang X, Mégevand P, Mercier MR, Lado FA, Mehta AD, Honey CJ (2017) iELVis: an open source MATLAB toolbox for localizing and visualizing human intracranial electrode data. J Neurosci Methods 281:40-48.

Guerrini R, Dobyns WB (2014) Malformations of cortical development: clinical features and genetic causes. Lancet Neurol 13:710-726.

Jacobs J, Zijlmans M, Zelmann R, Chatillon CE, Hall J, Olivier A, Dubeau F, Gotman J (2010) High-frequency electroencephalographic oscillations correlate with outcome of epilepsy surgery. Ann Neurol 67:209-220.

Kakita A, Hayashi S, Moro F, Guerrini R, Ozawa T, Ono K, Kameyama S, Walsh CA, Takahashi H (2002) Bilateral periventricular nodular heterotopia due to filamin 1 gene mutation: widespread glomeruloid microvascular anomaly and dysplastic cytoarchitecture in the cerebral cortex. Acta Neuropathol 104:649-657.

Katzner S, Nauhaus I, Benucci A, Bonin V, Ringach DL, Carandini M (2009) Local origin of field potentials in visual cortex. Neuron 61:35-41.

Keller CJ, Bickel S, Honey CJ, Groppe DM, Entz L, Craddock RC, Lado FA, Kelly C, Milham M, Mehta AD (2013) Neurophysiological investigation of spontaneous correlated and anticorrelated fluctuations of the BOLD signal. J Neurosci 33:6333-6342.

Kiebel SJ, Friston KJ (2004) Statistical parametric mapping for event-related potentials: I. Generic considerations. Neuroimage 22:492-502.

Kirschstein T, Fernández G, Grunwald T, Pezer N, Urbach H, Blümcke I, Van Roost D, Lehnertz K, Elger CE (2003) Heterotopias, cortical dysplasias and glioneural tumors participate in cognitive processing in patients with temporal lobe epilepsy. Neurosci Lett 338:237-241.

Kothare SV, VanLandingham K, Armon C, Luther JS, Friedman A, Radtke RA (1998) Seizure onset from periventricular nodular heterotopias: depth-electrode study. Neurology 51:1723-1727.

Kucyi A, Parvizi J (2020) Pupillary dynamics link spontaneous and taskevoked activations recorded directly from human insula. J Neurosci 40:6207-6218.

Kucyi A, Schrouff J, Bickel S, Foster BL, Shine JM, Parvizi J (2018) Intracranial electrophysiology reveals reproducible intrinsic functional connectivity within human brain networks. J Neurosci 38:4230-4242.

Kucyi A, Daitch A, Raccah O, Zhao B, Zhang C, Esterman M, Zeineh M, Halpern CH, Zhang K, Zhang J, Parvizi J (2020) Electrophysiological dynamics of antagonistic brain networks reflect attentional fluctuations. Nat Commun 11:325.

Li LM, Dubeau F, Andermann F, Fish DR, Watson C, Cascino GD, Berkovic SF, Moran N, Duncan JS, Olivier A, Leblanc R, Harkness W (1997) Periventricular nodular heterotopia and intractable temporal lobe epilepsy: poor outcome after temporal lobe resection. Ann Neurol 41:662668.

Liu S, Parvizi J (2019) Cognitive refractory state caused by spontaneous epileptic high-frequency oscillations in the human brain. Sci Transl Med 11: eaax7830.

Liu S, Sha Z, Sencer A, Aydoseli A, Bebek N, Abosch A, Henry T, Gurses C, Ince NF (2016) Exploring the time-frequency content of high frequency oscillations for automated identification of seizure onset zone in epilepsy. J Neural Eng 13:026026.

Liu S, Gurses C, Sha Z, Quach MM, Sencer A, Bebek N, Curry DJ, Prabhu S, Tummala S, Henry TR, Ince NF (2018) Stereotyped high-frequency oscillations discriminate seizure onset zones and critical functional cortex in focal epilepsy. Brain 141:713-730. 
Liu W, An D, Tong X, Niu R, Gong Q, Zhou D (2017) Region-specific connectivity in patients with periventricular nodular heterotopia and epilepsy: a study combining diffusion tensor imaging and functional MRI. Epilepsy Res 136:137-142.

Manning JR, Jacobs J, Fried I, Kahana MJ (2009) Broadband shifts in local field potential power spectra are correlated with single-neuron spiking in humans. J Neurosci 29:13613-13620.

Maris E, Oostenveld R (2007) Nonparametric statistical testing of EEG- and MEG-data. J Neurosci Methods 164:177-190.

McRae JF, Clayton S, Fitzgerald TW, Kaplanis J, Prigmore E, Rajan D, Sifrim A, Aitken S, Akawi N, Alvi M, Ambridge K, Barrett DM, Bayzetinova T, Jones P, Jones WD, King D, Krishnappa N, Mason LE, Singh T, Tivey AR, et al. (2017) Prevalence, phenotype and architecture of developmental disorders caused by de novo mutation. Nature 542:433-438.

Mirandola L, Mai RF, Francione S, Pelliccia V, Gozzo F, Sartori I, Nobili L, Cardinale F, Cossu M, Meletti S, Tassi L (2017) Stereo-EEG: diagnostic and therapeutic tool for periventricular nodular heterotopia epilepsies. Epilepsia 58:1962-1971.

Nir Y, Mukamel R, Dinstein I, Privman E, Harel M, Fisch L, Gelbard-Sagiv H, Kipervasser S, Andelman F, Neufeld MY, Kramer U, Arieli A, Fried I, Malach R (2008) Interhemispheric correlations of slow spontaneous neuronal fluctuations revealed in human sensory cortex. Nat Neurosci 11:1100-1108.

Oostenveld R, Fries P, Maris E, Schoffelen J-M (2011) FieldTrip: open source software for advanced analysis of MEG, EEG, and invasive electrophysiological data. Comput Intell Neurosci 2011:156869.

Papademetris X, Jackowski MP, Rajeevan N, DiStasio M, Okuda H, Constable RT, Staib LH (2006) BioImage suite: an integrated medical image analysis suite: an update. Insight J 2006:209.

Parvizi J, Kastner S (2018) Promises and limitations of human intracranial electroencephalography. Nat Neurosci 21:474-483.

Pizzo F, Roehri N, Catenoix H, Medina S, McGonigal A, Giusiano B, Carron R, Scavarda D, Ostrowsky K, Lepine A, Boulogne S, Scholly J, Hirsch E, Rheims S, Bénar CG, Bartolomei F (2017) Epileptogenic networks in nodular heterotopia: a stereoelectroencephalography study. Epilepsia 58:2112-2123.

Rosenberg M, Noonan S, DeGutis J, Esterman M (2013) Sustaining visual attention in the face of distraction: a novel gradual-onset continuous performance task. Atten Percept Psychophys 75:426-439.

Spreer J, Martin P, Greenlee MW, Wohlfarth R, Hammen A, Arnold SM, Schumacher M (2001) Functional MRI in patients with band heterotopia. Neuroimage 14:357-365.

Tassi L, Colombo N, Cossu M, Mai R, Francione S, Lo Russo G, Galli C, Bramerio M, Battaglia G, Garbelli R, Meroni A, Spreafico R (2005) Electroclinical, MRI and neuropathological study of 10 patients with nodular heterotopia, with surgical outcomes. Brain 128:321-337.

Thompson SA, Kalamangalam GP, Tandon N (2016) Intracranial evaluation and laser ablation for epilepsy with periventricular nodular heterotopia. Seizure 41:211-216.

Valton L, Guye M, McGonigal A, Marquis P, Wendling F, Régis J, Chauvel P, Bartolomei F (2008) Functional interactions in brain networks underlying epileptic seizures in bilateral diffuse periventricular heterotopia. Clin Neurophysiol 119:212-223.

van den Heuvel MP, Hulshoff Pol HE (2010) Exploring the brain network: a review on resting-state fMRI functional connectivity. Eur Neuropsychopharmacol 20:519-534.

Villani F, Vitali P, Scaioli V, Rodriguez G, Rosa M, Granata T, Avanzini G, Spreafico R, Angelini L (2004) Subcortical nodular heterotopia: a functional MRI and somatosensory evoked potentials study. Neurol Sci 25:225-229.

Wagner J, Elger CE, Urbach H, Bien CG (2009) Electric stimulation of periventricular heterotopia: participation in higher cerebral functions. Epilepsy Behav 14:425-428.

Watson BO, Ding M, Buzsáki G (2018) Temporal coupling of field potentials and action potentials in the neocortex. Eur J Neurosci 48:2482-2497.

Worrell GA, Jerbi K, Kobayashi K, Lina JM, Zelmann R, Le Van Quyen M (2012) Recording and analysis techniques for high-frequency oscillations. Prog Neurobiol 98:265-278. 